\title{
Low solar elongation searches for NEO: a deep sky test and its implications for survey strategies
}

\author{
Andrea Boattini ${ }^{1}$, A. Milani ${ }^{2}$, G. F. Gronchi ${ }^{2}$, T. Spahr ${ }^{3}$ \\ and G. B. Valsecchi ${ }^{4}$ \\ ${ }^{1}$ Department of Physics, University of Tor Vergata, and OAR-INAF, Via Frascati 33, 00040 \\ Monteporzio Catone (Roma), Italy, email: boattini@iasf-roma.inaf.it \\ ${ }^{2}$ Department of Mathematics, University of Pisa, Piazza Pontecorvo 5, 56127 Pisa, Italy \\ email: milani@dm.unipi.it \\ ${ }^{3}$ Minor Planet Center, Smithsonian Astrophysical Observatory, 60 Garden Street, \\ Cambridge, MA 02138, USA \\ ${ }^{4}$ IASF-INAF, Via Fosso del Cavaliere 100, 00133 Roma, Italy
}

\begin{abstract}
A survey for NEO aiming at 90\% completeness for a given size range cannot ignore that a significant fraction of the population is observable essentially only at low solar elongation, in the so called "sweet spots". There are several penalties for such low elongation: poorer observing conditions imply a lower limiting magnitude, shorter available time in each night and a more difficult orbit determination. Our aim is to show that these difficulties can be overcome. We have tested the observation procedures and the mathematical methods of orbit determination on two sweet spot test runs. One was performed at ESO La Silla in Jan-Feb 2005, the other at Mauna Kea in Sept-Dec 2005. The results of the tests are presented in this paper; the observed area was not large enough (especially at Mauna Kea) to discover a significant number of new NEO, the purpose was rather to identify the problems. These tests have allowed us to identify all the key elements to be accounted for in the strategy for a successful sweet spot NEO survey. When very short arc observations from different nights have to be identified, a specific difficulty occurs at the sweet spots: the same set of observations from three nights can be fitted to two incompatible orbits, in most cases including one NEO and one MBA. This can lead to two different failures (false positive, false negative) in deciding whether a NEO has been discovered. The classical theory of preliminary orbits shows that three observations at an elongation less than $116.5^{\circ}$ can be compatible with two different orbits. From this theory we have derived an algorithm to find the alternate solution, if it exists, when only one is available. In this way we have generated a set of examples of possible discoveries with two well determined but incompatible solutions. Most of the MBA-NEO alternatives have been solved by finding a known MBA which could be identified; in two cases the MBA solution has been confirmed by a later observation.
\end{abstract}

Keywords. surveys, orbit determination, identification

\section{Purpose and Method}

A survey for NEOs aiming at $90 \%$ completeness for a given size range cannot ignore that a significant fraction of the population passes in the neighborhood of opposition either never or very rarely or only in very poor observing conditions (Stokes et al. 2003). This implies that there is a bias against the discovery of Inner Earth Objects (IEO), Atens and other NEOs with larger eccentricity and/or inclination.

To compensate for this bias a fraction of the available telescope time needs to be used at low solar elongation (around or below $90^{\circ}$ ), in the so called sweet spots. Many potential Earth impactors can be discovered only by scanning the sweet spots 
(Chesley \& Spahr 2002). Indeed, observing this part of the sky - that is along the Earth's orbit - with powerful telescopes and appropriate orbital discrimination and calculation tools can yield a large number of Potentially Hazardous Asteroids (PHA). A good example of this is the discovery at a sweet spot of (99942) Apophis, which so far is the best and more challenging example for impact calculation studies.

We have studied the observing strategy and data processing methods which could be used to implement such a sweet spot survey, either as a separate program or as a subset of a next generation survey. The specific difficulties of such an approach are:

1) Lower limiting magnitude at the sweet spots (for the same telescope and camera) than near opposition, due to larger airmass, poorer seeing and higher sky background.

2) Rapid variability of the observing conditions and shorter total time available for observations in each night.

3) Larger average dispersion in proper motion, making discrimination between NEOs and Main Belt Asteroids (MBAs) more difficult.

4) Orbit Determination subject to problems from double and multiple solutions, from false identifications and computational overload.

Difficulty 1) can be overcome with adequate equipment at a high quality observing site. For 2) we propose using a short observing time each night, near sunrise and/or sunset, for many nights, exploiting the lower competition for telescope time under conditions inappropriate for other programs. We also use a short Transient Time Interval (TTI) between frames covering the same area (e.g., 15 minutes) to get more similar conditions.

Difficulty 3) can be overcome if the survey covers a large enough area to follow up automatically most objects, including most NEOs, as will be the case for the next generation all sky surveys. For small field surveys, targeted follow up is practically impossible if discrimination of NEOs is not achieved from the very first detections. As we will see, this is the main difficulty of a small scale test such as the one presented below. On the other hand, our purpose was not to obtain a number of discoveries comparable to large field of view surveys, which is anyway impossible, but to obtain enough discoveries and recoveries to validate the procedures.

Difficulty 4) was not enough considered in previous discussions of the sweet spot strategies; we will address this in detail in Section 2.

The difficulties seen above are somewhat compensated by significant advantages.

i) comparatively small NEOs can be detected at the achievable limiting magnitudes; the same objects would be either not observable or much dimmer near opposition.

ii) the MBAs are much dimmer than they are at opposition: this implies that most MBAs imaged at the sweet spots are already known, a prior information which is very effective in discriminating from NEOs.

iii) distant bodies, like Trans Neptunians, move so slowly that confusion is unlikely.

iv) even if the total number of discoveries in the sweet spots is lower than in a comparable telescope time at opposition, the number of interesting objects (Atens, IEOs, PHAs, even Virtual Impactors like Apophis) is very significant.

This paper discusses the specific orbit determination problems and their solution, presents our test sweet spot survey and discusses the result, allowing us to draw conclusions on the strategy to be used for future operational surveys.

\section{Identification and Orbit Determination Problems}

The problems of orbit determination occurring in a sweet spot (sub)survey are three: multiple, or anyway weakly determined, solutions; double solutions; false identifications. 


\subsection{Weakly determined solutions}

Even after accumulating observations of the same object over several nights, the orbit may be weakly determined, because the observed arc is still too short. In such cases there is typically a weak direction (in orbital elements space) along which the RMS of the residuals increases very little even by moving very far from the nominal, minimum RMS solution. As described in (Milani et al. 2005a), it is possible to define a continuous line of orbital elements, the Line Of Variations (LOV), containing constrained least squares solutions, in intuitive terms solutions with just 5 free parameters and a sixth parameter fixed at an arbitrary value (the one along the weakly constrained direction).

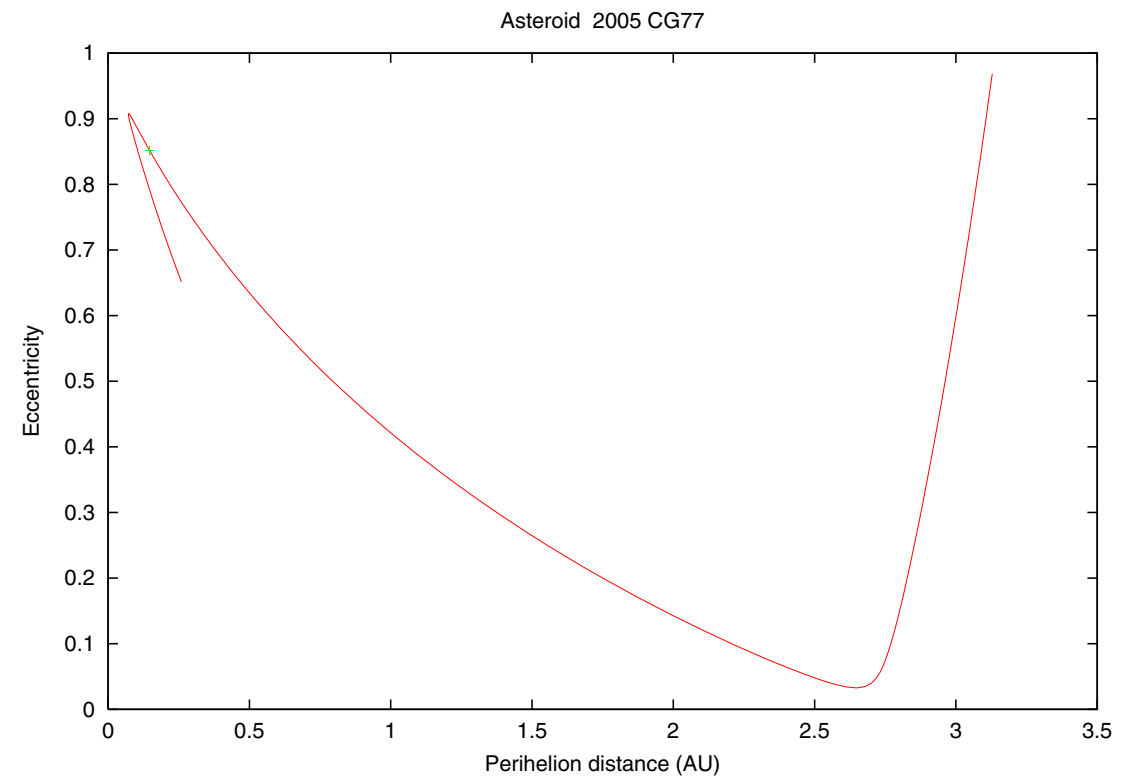

Figure 1. For $2005 \mathrm{CG}_{77}$ eccentricity and peculiar distance of the solutions along the LOV. Note that all the solutions in this plot correspond to residuals with a RMS less than 0.25 arcsec, as shown in Figure 4. Given that low eccentricity MBA solutions are possible, most likely they are the real ones, but there is no way to draw such a conclusion based on the available observations only.

The changes of the elements along the LOV can be extreme, as in the example of $2005 \mathrm{CG}_{77}$, one of the asteroids discovered in our test survey, with observation in three consecutive nights. As shown by the plot of 600 LOV solutions in Figure 1, there are solutions fitting in a satisfactory way the observations corresponding to MBA, NEO and even comet-like orbits (not shown, along the continuation of the LOV on the right of the figure). Targeted recovery in the next opposition would have required an inordinate use of telescope resources; as a matter of fact, it has not been recovered. The set of 10 observations in 3 nights forms an observed arc of type 2, according to the definition by (Milani et al. 2006), that is, the information contained in the data is equivalent to two arcs each without significant curvature (aligned, within the astrometric error, along a great circle). Roughly speaking, this 3-night orbit is not significantly better than in the case of two well separated nights of observation.

Indeed, all the cases in which an object has been observed only in two nights are of the same type, with largely undetermined orbit. In our opinion this type of designations 
should not be considered discoveries. The conclusion relevant for planning surveys is that the use of consecutive nights on the same area has to be avoided.

\subsection{Double solutions}

The classical theory of preliminary orbits (methods of Laplace and Gauss) shows that three observations at an elongation less than $116^{\circ} .5$ can be compatible with two different orbits, depending upon the values of elongation, distance and curvature of the observed arc. The classical analysis of the problem, based on the solutions of a degree 8 polynomial equation (Plummer 1918; Danby 1989), allows us to describe the behavior of the preliminary orbit algorithms in a plane with polar geocentric coordinates $(\rho, \epsilon)$ (with $\rho=$ distance from Earth, $\epsilon=$ solar elongation). Note that this theory uses the approximation neglecting the topocentric correction.

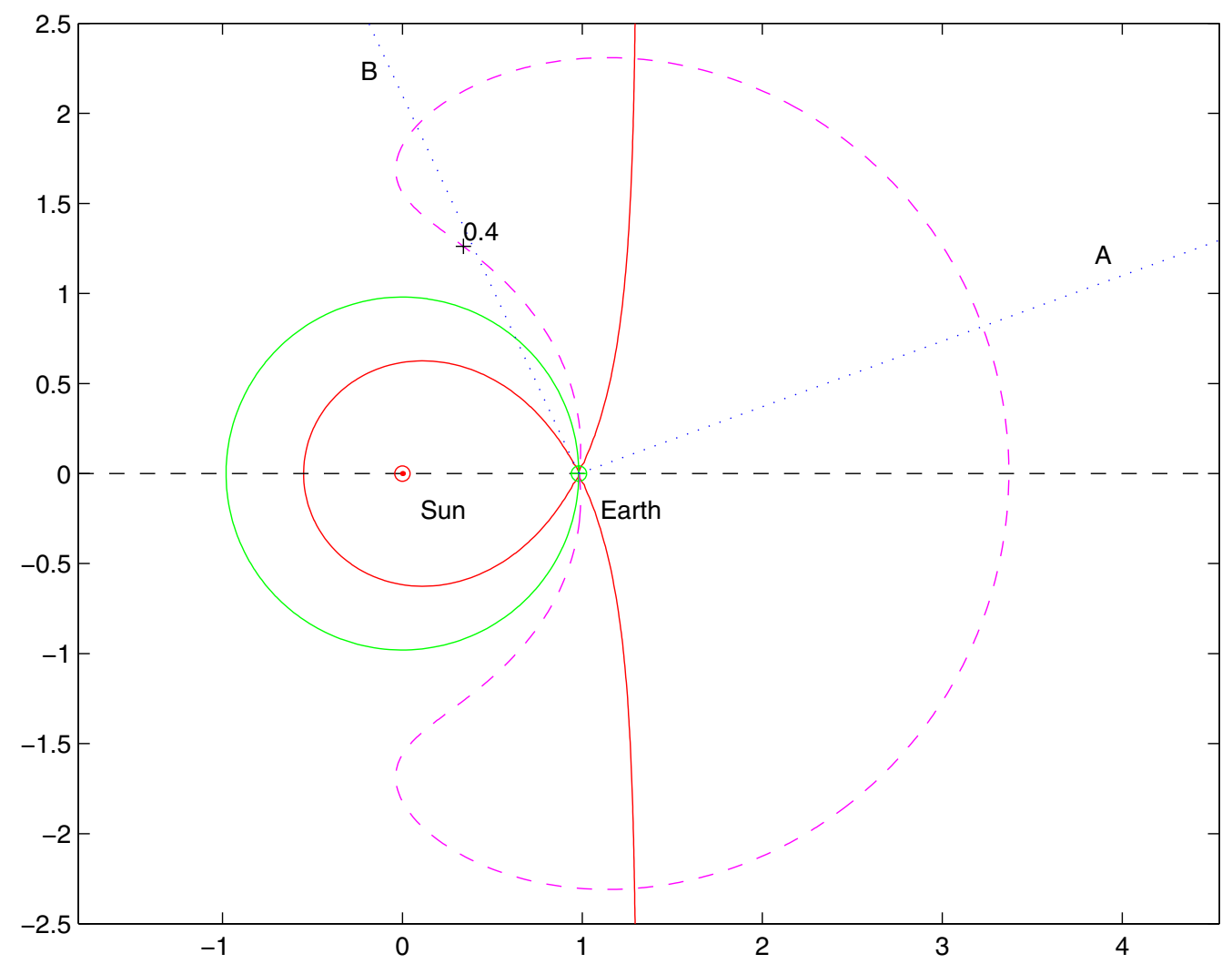

Figure 2. The solutions of the first approximation equation for preliminary orbits, given either three observations (method of Gauss) or the observations with their first and second derivatives (method of Laplace). When observing near opposition (line A: elongation $160^{\circ}$ ) there can be only one solution, while in the sweet spots (line B: elongation $65^{\circ}$ ) there can be two solutions, as well as none if the direction and curvature are incompatible.

Figure 2 shows as continuous lines the boundary between the regions with 1 and with either 0 or 2 solutions. The actual solutions for a given elongation and curvature appear in the figure as intersections of a dotted line representing the direction of observation with a dashed level line of a quantity related to curvature. Double solutions can occur at $|\epsilon| \leqslant 116^{\circ} .5$, on the left of the loop-shaped limiting curve and outside of the zero curvature circle with radius equal to the Sun-Earth distance. Inside the circle and the loop there 


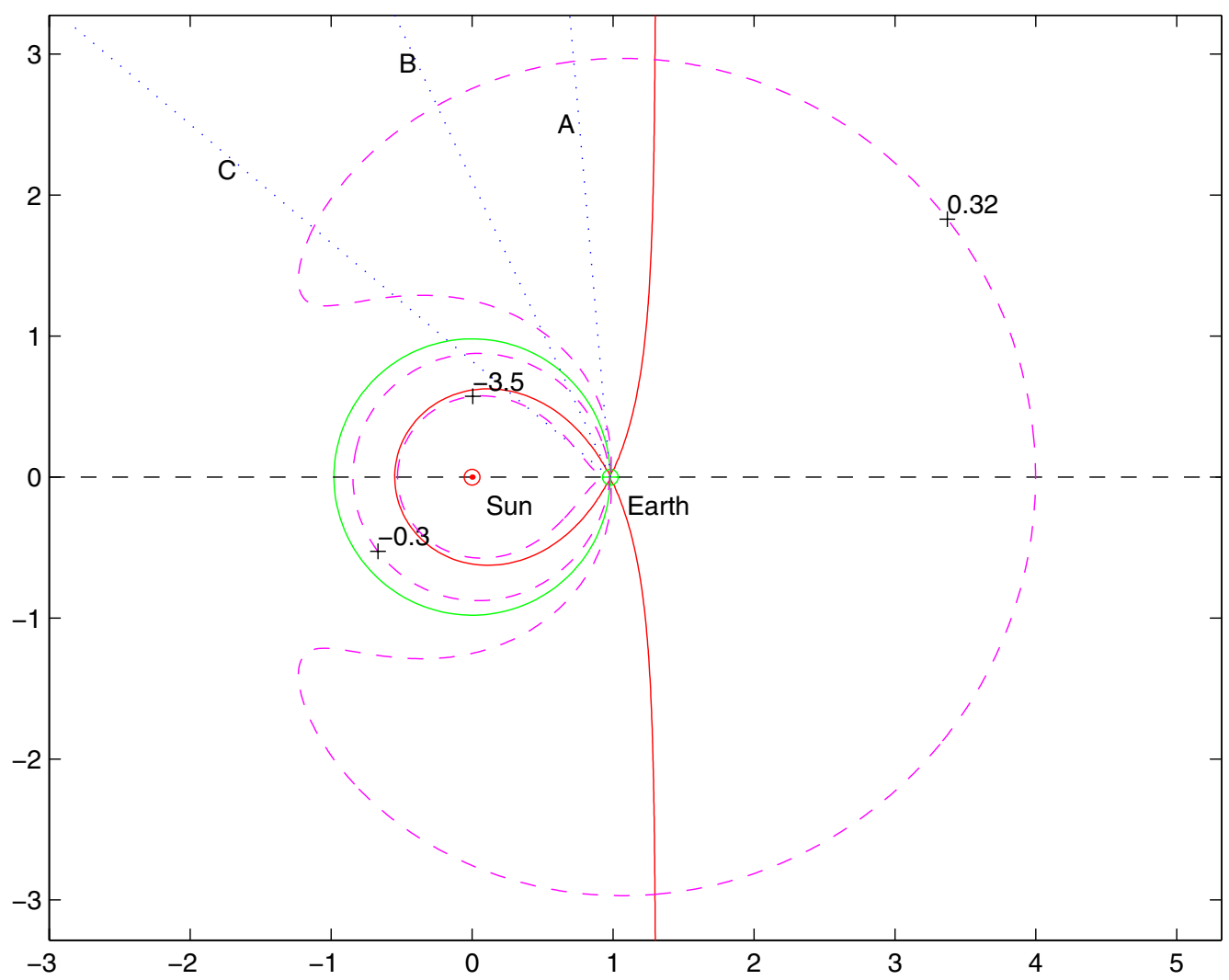

Figure 3. As in the previous Figure, showing a double solution near quadrature (line A: elongation $85^{\circ}$ ). The possibility of both single and double solutions depending upon the value of curvature is shown for line $\mathrm{B}$ (elongation $65^{\circ}$ ) and line $\mathrm{C}\left(\right.$ elongation $40^{\circ}$ ).

is another region with double solution, which can be reached only for $|\epsilon| \leqslant 63^{\circ} .5$ : these very low elongations are practically reachable only from very good sites like Mauna Kea. For $|\epsilon| \leqslant 90^{\circ}$ both one and two solutions are possible, depending upon the curvature, that is upon the actual heliocentric distance (see Figure 3).

The double solutions for the preliminary orbit, when used as first guess for a standard differential correction procedure, become double local minima of the target function (weighed sum of squares of the residuals) (See Milani et al. 2005a, Figure 11). If there are only three nights of observations, with a short time span for each night (as it is unavoidable for low elongation), the two minima are low and both correspond to acceptable solutions; if there are data over more than three nights and/or the data in each night have a sufficient time span and good accuracy, one of the two minima corresponds to a much higher RMS and it may be possible to discard it.

As an example, the least squares fit for $2005 \mathrm{CG}_{77}$ has two minima corresponding to an Apollo orbit with RMS 0.069 arcsec and an Aten orbit with RMS 0.126 arcsec. Not only both are acceptable fits, but also the solutions along the LOV in between the two and well beyond are acceptable, as is shown by Figure 4. For better determined discoveries, e.g., with arcs of type 3 , only two small segments along the LOV near the two local minima have acceptable RMS.

The most embarrassing case for surveys is when one of the two least squares solutions is a NEO orbit and the other is of MBA type, which happens very often. Then it is not 


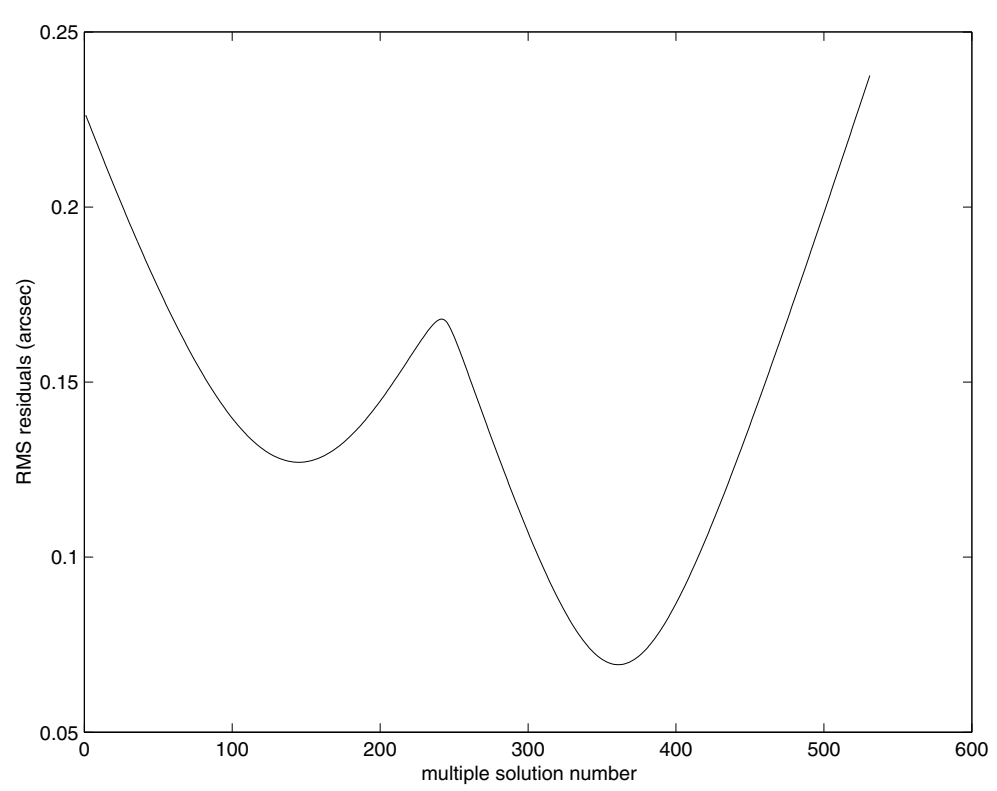

Figure 4. For the object $2005 \mathrm{CG}_{77}, 600$ alternate orbit solutions along the Line Of Variations have been computed. The RMS of the residuals ( $\operatorname{arcsec})$ is plotted against the solution number, showing two local minima.

possible to conclude that the object is a NEO; actually, because of known population statistics in most cases it is an MBA. On the other hand, it is not possible to exclude that it is a NEO, and by not following it up the risk is taken of missing an important discovery, defeating the very purpose of a sweet spot survey. If the object actually is a NEO, it may not pass at the opposition (under possible observing conditions) in the same year and for a long time. The radical solution is to obtain 4 well spaced nights of observations.

\subsubsection{False identifications}

To avoid weakly constrained orbits, the interval between nights observing the same area should be increased. On the other hand, this increases the likelihood of false identifications between short arcs of different nights. In fact, two night identifications with well separated nights are so unreliable to be useful almost only as intermediary step for finding 3 nighters (Milani et al. 2005b). Surprisingly, even 3 night identifications can be false and be fit with very small residuals and a well determined, spurious orbit.

The most damaging property of these spurious orbits is that they are very often spurious NEOs. Actually, in our test all the 3-night identifications we found and which we could prove false had only NEO type orbits, and were formed with data from two different MBAs. We do not have a formal proof that this must always be the case. Without a method to dismiss these false identifications, we would be forced to waste resources in follow up activities bound to fail.

The conclusion is that it is necessary to select an interval between the nights of observations of the same area which is of intermediate duration. The optimal value would depend upon the achievable limiting magnitude (for deeper surveys the number of false identifications grows with the square of the detections number density) and upon the astrometric accuracy (the better the astrometry, the longer the useful interval). The upper limit is set by the need to have at least 4 nights of observation in the same lunation. 


\section{The experimental survey}

We have tested the observation procedures and the mathematical methods of identification/orbit determination on two sweet spot test runs. One was performed at ESO La Silla in Jan-Feb 2005, using the Wide field Imager on the $2.2 \mathrm{~m}$ telescope (field of view $34 \times 33$ arcmin, pixel scale in $3 \times 3$ binning mode 0.71 arcsec); for the use of this instrument, see (Boattini et al. 2004). This test covered 181 square degrees at low elongation over the first two and last two hours of the observing night, in two runs of 10 nights each in January and February. Observations began 20 minutes before the end of the astronomical twilight, and ended symmetrically after the start of twilight. The observation were unfiltered with $60 \mathrm{~s}$ exposure, and the limiting magnitude practically achieved was equivalent to 21.5 in the $\mathrm{R}$ band. The astrometric accuracy was about $0.2 \operatorname{arcsec}$ in all nights.

With the goal of probing even deeper limiting magnitudes, we made a second test at Mauna Kea in 7 nights of Sept-Dec 2005, using the MegaCam on the CFHT $3.6 \mathrm{~m}$ telescope with an R filter and $60 \mathrm{~s}$ exposure. The MegaCam is a mosaic of $402048 \times 4612$ pixel CCDs, with a field of view of $1 \times 1$ degree. We even used a portion of one night of SupremeCam at the Subaru $8.3 \mathrm{~m}$ (field of view $34 \times 27$ arcmin). For both telescopes the pixel scale in $2 \times 2$ binning mode was 0.37 arcsec and the time used was only the last 20 minutes before the start of the astronomical twilight. The total coverage at low elongation was 35 square degrees over 8 nights, with limiting magnitude equivalent to 22.5 in the $\mathrm{R}$ band. The astrometric accuracy was particularly good, with a random component below 0.1 arcsec. The astrometric catalog used was USNO-B1.0 (Monet et al. 2003); systematic effects at this level of accuracy are difficult to assess.

The data were processed in near real time only to look for the fast moving objects. The complete astrometric reduction was performed offline. The data was then passed through the identification and orbit determination computer code which has been developed, within the OrbFit system, for the simulations of the Pan-STARRS survey; for the algorithms see (Milani et al. 2004; Milani et al. 2005b). However, additional code had to be written to handle the problem of double solutions of Section 2.2; the same code has been later retrofitted to the Pan-STARRS simulation code.

The next processing step has required a significant interaction with the Minor Planet Center (MPC). Of course, a large survey would only need to compare with its own observations near opposition, to recognize the known MBAs among the observations near quadrature. For a small survey it is essential to consult the largest possible database of known asteroids. We have used for this purpose the MPChecker online servicet. In some difficult cases, the interaction with the MPC databases was more complicated and will be described in the next Section.

\section{Results}

\subsection{The La Silla test}

The observational data from La Silla amounted to a total of more than 5,000 detections of moving objects. From the point of view of the observation strategy, the results from La Silla were affected by two problems:

- In the January 2005 run, most of the objects detected and measured were observed only once, with the purpose of covering "fresh sky" as much as possible. During the run in February 2005 we revisited the same fields a number of times, looking for identifications.

- Even when the observed arcs included more than one night (mostly in the February

$\dagger$ http://scully.harvard.edu/cgi/CheckMP 
run) the total time span was short, usually 2-4 days; this was due to the short total duration of the run. The good side of short time spans is that the identifications from different nights turned out to be all correct (that is, they belong to the same object) apart from just one case.

Thus the results of January are based on the traditional method of following up the fast moving objects, and indeed a new NEO was found in this way. The February run was more interesting for the development of a new sweet spot surveying strategy. Table 1 shows the results from the test at La Silla, essentially only the February run. The cases listed are only those in which a NEO solution was possible; some objects with only Main Belt solutions were also detected.

Table 1. Summary of the objects detected at La Silla exhibiting multiple orbit solutions. Long-LOV indicates cases in which the multiple solutions along the Line-Of-Variations (LOV) span different orbital type (NEA, MBA, Trojan, etc.). In addition to those shown, there were 6 cases of single orbit solution, 4 of which correspond to known MBAs, 1 to a new Trojan, and the last was found to be a false identification.

\begin{tabular}{ccc}
\hline Observations & Multiple solutions & Identification \\
\hline 4 nights & 2 (MBA-NEA) & 2 known MBAs \\
\hline 3 nights & 13 (MBA-Aten) & 12 known MBAs +1 new MBA \\
& 14 long LOV & 10 known MBAs +1 new MBA \\
\hline
\end{tabular}

Although the results are negative, in that no new NEO moving as main belters was discovered, this test pointed out the importance of the double and multiple solutions problem (Table 1). There were 13 cases of 3 -nighters with double solutions, including one NEO; the same even in 2 cases of 4-nighters. There were 14 long LOV solutions, too weak to exclude a NEO solution.

In 24 out of these 29 cases the problem was solved by recognizing the detected object as a known MBA (with MPChecker). Two other cases (one double solution and one long LOV) have been solved by recovery and identification. In three cases a designation was assigned by the MPC: $2005 \mathrm{CL}_{61}, 2005 \mathrm{DE}_{3}$ and $2005 \mathrm{CG}_{77}$ (see Figure 1), but the objects have been neither recovered nor identified yet, thus strictly speaking we do not yet know if they are NEOs, although of course they are more likely to be MBAs.

\subsection{The Mauna Kea test}

At Mauna Kea we were able to fully implement a new observing strategy and distribute the observations over an arc of about 30-40 days; in this way, we could obtain many identifications and good orbits from the $\simeq 3,700$ detections of moving objects. Weak solutions, with long LOV not allowing us to discriminate NEOs from MBAs, did not occur in any 3-night orbit, because of the longer time spans. Nevertheless, the number of nights of observations was not enough to solve all the problems of double solutions and false identifications.

In this observation run we intentionally included a known Aten (1998 XE 12 ), with an apparent motion compatible with an MBA, especially in the first two nights (proper motion $0^{\circ} .49 / \mathrm{d}$ and $0^{\circ} .52 / \mathrm{d}$; the third night had $0^{\circ} .60 / \mathrm{d}$, which is indicative of a nearer object. The identification and orbit determination software had no problem in finding two solutions, both of NEA type (one Aten and one Apollo).

Table 2 shows the results from the run at Mauna Kea. There were 16 cases with double solutions, all with very good fits (maximum RMS of the residuals was 0.10 arcsec) and 
Table 2. Summary of the objects detected at Mauna Kea exhibiting multiple orbit solutions. In addition to those shown, there were 5 cases of single orbit solution for detection over 4 nights, 3 of which correspond to known MBAs, and 2 to new MBAs, and 13 cases of single orbit solution for detection over 3 nights; all of these come from false linking of uncorrelated detections, and in most cases correspond to a NEA orbit solution.

\begin{tabular}{ccc}
\hline Observations & Multiple solutions & Identification \\
\hline 3 nights & 16 (MBA-NEA) & 15 known MBAs +1 new MBA \\
& 1 (NEA-NEA) & known Aten inserted on purpose \\
\hline
\end{tabular}

with one MBA and one NEA solution. The special case of $1998 \mathrm{XE}_{12}$ had RMS of 0.23 arcsec (before removing the outlier) for the best NEA solution.

Using MPChecker we were able to solve 15 out of these cases, identifying them with known MBAs. There were no false identifications among these.

In one case, no identification with known MBAs was found, and the designation 2005 $\mathrm{SW}_{277}$ was assigned by the MPC. Even if MBAs are on average brighter by two magnitudes at opposition, a sweet spot survey with limiting magnitude 22.5 may occasionally find an MBA not yet discovered by the present surveys. However, it was later possible to attribute some LONEOS observations from a single night (taken four months later) to $2005 \mathrm{SW}_{277}$; the 4-night orbit was uniquely determined and was of type MBA.

Besides the results listed in Table 2, the OrbFit software proposed 13 additional 3night identifications with a single orbital solution and RMS of the residuals between 0.14 and 0.24 arcsec. The solutions with RMS $>0.24$ arcsec have been automatically discarded. In all these 13 cases it was possible to identify at least one of the 3 nights with a known MBA, in some cases 2-3 nights with different MBAs, thus confirming that all these proposed identifications were false.

The result which may appear disturbing is that in 13 out of 13 cases the spurious solutions were NEO type orbits. Moreover, the reason why there was no double solution was, in 10 cases out of 13 , that the preliminary orbit was also unique (in 2 cases the preliminary orbit algorithm gave 0 solutions, in 1 case there were 2 solutions). The sample is too small to draw statistically significant conclusions, but it is enough to suggest some correlation between the false identification and the orbital class.

A qualitative explanation can be as follows. If three single night arcs are wrongly identified, it is very unlikely that they are well aligned along a single great circle, thus the curvature of the whole observed arc will be comparatively large. The curvature goes to $-\infty$ as the heliocentric distance goes to zero. Thus, if the sign happens to be right, a large spurious curvature can be interpreted by the preliminary orbit algorithm as if the object is closer to the Sun than the Earth, and this implies a NEO orbit. Moreover, if the elongation is more than $63^{\circ} .5$, the objects belongs to the region where there is a single solution (see Section 2.2 and Figure 3).

Thus the problem of false identifications is especially critical at the sweet spots, because it can result in false positive NEO discoveries. The other problem, the double solutions, can result in false negatives unless both solutions are reliably computed.

\section{Conclusions}

We have performed a small, but complete test of a sweet spot survey. We have identified the specific difficulties, including the ones occurring in identification and orbit determination which previously had not been investigated. In particular, the weakly determined (long LOV) solutions can be avoided by properly spacing the observing nights. We have 
not determined an optimal spacing, for now we can only conclude that it should be between 2 and 6 days.

A difficulty not found near opposition is the occurrence of double solutions. A possible remedy is to exploit the prior knowledge of the MBAs observable in the sweet spots; this is possible if the sweet spot survey is a component of a larger survey covering also the opposition region up to the same limiting magnitude. Another solution is scheduling the sweet spot survey to guarantee at least 4 nights of observations; this is possible because of the reduced competition for telescope time at times near the astronomical twilight.

The third difficulty, which occurs also near opposition, is due to false identifications. In the sweet spots, a false identification often results in spurious NEO orbits, and such false positives could be the source of a waste of astronomical resources. If the false identifications combine observations of different MBAs, again with enough prior information collected at opposition these spurious cases can be discarded, as it happened in our test. However, we cannot exclude that in a larger experiment this problem could lead to dubious cases.

The problems with the observation strategy and scheduling are very severe for a survey covering a small area in the sky, while they are easy to solve for the wide field surveys and when dedicated follow up resources are available. What needs to be investigated is a possible strategy for a narrow field survey going much deeper than the next generation all sky surveys.

\section{Acknowledgements}

This research has been funded by: the Italian Ministero dell'Università e della Ricerca Scientifica e Tecnologica, PRIN 2004 project "The Near Earth Objects as an opportunity to understand physical and dynamical properties of all the solar system small bodies". The observations from Mauna Kea were taken within the UHAS survey, in collaboration with D. Tholen and F. Bernardi; the ones from La Silla were made possible by allocation of telescope time by the standard ESO procedure. We thank D. Tholen, F. Bernardi, N. Kaiser, R. Jedicke, L. Denneau and O. Hainaut for useful discussions and suggestions.

\section{References}

Boattini, A., et al. 2004, Astron. Astrophys., 418, 743

Chesley, S. \& Spahr T., 2002, in NASA Workshop on Scientific Requirements for Mitigation of Hazardous Comets and Asteroids, Arlington, VA, September 3-6, 2002

Danby, J. M. E. 1989, Fundamentals of Celestial Mechanics, Willmann-Bell, Richmond (VA)

Milani, A., Gronchi, G. F., de' Michieli Vitturi, M. \& Knežević, Z. 2004, Celest. Mech. Dyn. Astron. 90, 59

Milani, A., Sansaturio, M. E., Tommei, G., Arratia, O. \& Chesley, S. R. 2005a, Astron. Astrophys. 431,729

Milani, A., Gronchi, G. F., Knežević, Z., Sansaturio, M. E. \& Arratia, O. 2005b, Icarus, 179, 350

Milani, A., Gronchi, G. F. \& Knežević, Z. 2006, Earth, Moon and Planets, in press

Monet, D. et al. 2003, Astron. J., 125, 984

Plummer, H. C. 1918, An introductory treatise on dynamical astronomy, Dover Publications, New York.

Stokes, G. H., et al. 2003, Report of the Near-Earth Object Science Definition Team. August 22, 2003 (available at http://neo.jpl.nasa.gov/neo/report.html). 